\title{
A SECURE, ROBUST AND EFFECTIVE CRYPTOSCHEME USING DATA OBFUSCATION OF ENTITY DETECTION AND REPLACEMENT \& ENCRYPTION FOR ENSURING DATA PRIVACY IN PRIVATE CLOUD
}

\author{
D. Yakobu ${ }^{1}$, Kalluri Hemantha Kumar and Venkatesulu Dondeti ${ }^{2}$ \\ ${ }^{1,2}$ Department of Computer Science \& Engineering, \\ Vignan's Foundation for Science Technology and Research, \\ A.P, India-522213 \\ yakobdsp83@gmail.com, khkcsevignan@gmail.com, \\ drvenkatesulud43@gmail.com
}

\begin{abstract}
Cloud has been emerging, popular and very demanding technology now a day. Cloud has got wide popularity with its sophisticated features such as internet access, more storage, easy setup, automatic updates, low cost and resource provisioning based on "pay as you go" policy. In spite of advantages, security is considered to be more important and drew the attention of many researchers. The data storage is becoming an indispensable measurement in cloud and most of the times cloud does not guarantee that information/data that has been stored is secured from illegitimate access. Many researchers are working to ensure information security in cloud but unfortunately they do not provide adequate security. This paper is aiming to propose an efficient algorithm with obfuscation and cryptography for unstructured data. The algorithm works to preserve confidentiality of data stored in cloud at two levels. At the fist level the algorithm obfuscate the document by replacing the key words (obfuscation), and at the second level obfuscated document is encrypted using traditional RSA algorithm for better security. Experimental results shows that the proposed algorithm yields good results.
\end{abstract}

\section{Keywords - Cloud Computing, Confidentiality, Obfuscation, Cryptography}

\section{INTRODUCTION}

Cloud computing is an emerging technology, and every organization wants to adopt this technology these days. It is cost effective and simple to use. The user pays as he uses the cloud resources [1]. Major beneficiaries are smaller enterprises and individual professional who develop the applications without having huge investments. It is affordable as pays for what he uses. This technology mainly works with three modules. One is cloud service provider [2], who places the data or resources into the cloud. Second module is cloud service consumer who accesses the required resources from cloud. And the third module is a web interface through which cloud user can place the resources in the cloud or access the services of the cloud [3]. These services can be categorized as Software as a service (SaaS), Infrastructure as a service (IaaS), Application as a service (AaaS), and Platform as a service (PaaS), Communication as a service, Network as a 
service and Database as a service etc. [4]. These services will be deployed in three models, such as through public, private and hybrid etc. [5] The cloud technology has three key features. They are pay-as-you go [5], flexibility, auto scaling [6], elasticity [7] etc. According to pay-as-you-go, the billing process is done based on the amount of utilization of the resources [39][40]. This feature is playing vital role for the rise of this technology [6].

In spite of having such greater features, it comes up with security issues [7]. The security issues are related to information security [8], network security, infrastructure security, confidentiality, attacks etc. [9][10]. The major research work focused on providing security to data in the cloud from being accessed by illegitimate users [11]. This is known as information/data security which is the subject of our work [12]. Information that is stored in cloud, should be kept confidential according to the requirements of the cloud user. Cloud computing still not guaranteed the information security [13][14]. Because of such problems in security, this technology is far away from banking field. Data that is stored/shared in cloud typically include customer transaction information, customer preferences, customer feedback and survey feedback and patients health information etc., [37][38]. Hence, applications in cloud computing research typically requires effective techniques/algorithms to preserve the privacy of cloud user's confidential information [15]. These techniques/algorithms should work effectively by properly processing, testing and validating the large amounts of data. Real life data which has most confidential information is most desirable for verifying and validating the effectiveness and suitability these techniques, algorithms and mechanisms.

Effective techniques and algorithms must be introduced to ensure the privacy of such customer information before it is made public [16][17]. Some techniques that scramble or replace the sensitive data or information in documents, in a manner that does not expose the sensitive or the confidential information. These techniques ensure that when documents are shared, the illegitimate user could not capture the user's personal information but enables the readability of data [18]. Other techniques includes cryptography, which converts the data in to some other form that can't be read and understand by anyone unless it is converted in to its original form [19][20]. In the first case, though it does not provides actual information, there is still a possibility of data breach since it allows the data readability. In the second case, though it does not enable the readability, there is a possibility that third party can decrypt the data and use it. Hence, techniques should be designed such that no confidential information is disclosed to the illegitimate user, but the document should be in a manner that other analytical process can be performed on the data [21][22][14].

In our work, we designed and implemented an algorithm for preserving the privacy of structured and unstructured data such as patient health information and company policies [41]. Our algorithm protects the data at two levels. At first level it replaces the original information with dummy information by identifying key words from the document, allows readability of the document and it is possible to recreate the original document. Retaining readability of document has few advantages: (i) when third party (illegitimate user) access get access to document, he can't conclude which data is original and which is replaced, (ii) since enough information available, we can perform analytical and processing application on document. At the second level it converts the sensitive information in to cipher form using traditional RSA technique to prevent readability. Experimental results shows that it yields the effective results.

\subsection{ORGANISATION OF THE PAPER}

The overview of this paper is as follows. Section II describes with the related works. Section III explains the working of proposed algorithm. Section IV provides experiments with proposed algorithm with sample data. Section V describes the results and explorations. Section VI ends in the conclusion. 


\section{LITERATURE SURVEY}

Bahare Hashemzade and Ali Maroosi [18], proposed a hybrid signal and encryption obfuscation method which uses a signal method to change the tree- and graph-like structure of the program into the star structure and hide the control flow graph of the problem. The disadvantage of signal method is high number of call \& return instructions. This examination recommended adding a dispatcher to the program that transforms the signal program to the control flow graph. This dispatcher was scrambled to keep it secure from illegitimate users. Besides, another methodology has been recommended to gauge complexity and strength. Five capacities were offered so as to compute the estimations of complexity and strength. The comparative results shows that the proposed method presents a performance advantage over existing methods.

Sreeram Viswanath Balakrishnan [23], proposed a method " Data Obfuscation of text data using entity detection and replacement" which process the document and entities coordinating the configuration parameters are labeled for obfuscation. The labeled elements are then Substituted in the document with jumbling content. The jumbling content can be gotten from a hash table. The hash table might be utilized to give a turnaround jumbling technique by which unique information can be reestablished to a obfuscated documented.

Leonardo H. Iwaya [24], presented a privacy-preserving ontology-based obfuscation mechanism intended to obfuscate health information either for primary or secondary use. On account of primary use, minimization of individual's information implies that an actor gets no more data than required and with a fitting semantic level. For secondary use, the proposed mechanism can limit the semantic loss of information, with the end goal that a high level of utility is kept up, while information is anonymised to the specified DA necessities.

Confidentiality technique using data obfuscation to enhance security of stored data in public cloud is proposed [25] by S. Arul Oli and Dr.L. Arockiam. This technique protects the numerical data in cloud storage. When the user wants to encrypt the inconsistent numerical data with confusion the proposed technology is flexible and compatible. This method is the symmetric cryptosystem. There are two keys used in this proposed algorithm for encryption and decryption. And two keys are integer values. With these two keys opacity of numerical data is possible through the proposed ARO_Obfus_CT to preserve data in the public cloud.

\section{PROPOSED METHODOLOGY}

The proposed algorithm aims at securing the structured/unstructured data such as patient health information and company policies that has been shared through public cloud [35][36]. It preserves the privacy of sensitive information. It secures data at two levels: At first level it replaces the original information with dummy information by identifying and replacing the key words in the document using NLP techniques [26][27] and allows readability of the document such that it is possible to recreate the original document. Preserving readability of document has few advantages: (i) when third party (illegitimate user) access get access to document, he can't conclude which data is original and which is replaced, (ii) since enough information available, we can perform analytical and processing application on document. At the second level it converts the sensitive information in to cipher form using traditional RSA technique to prevent readability. The Figure 1 shows detailed structure of algorithm. 


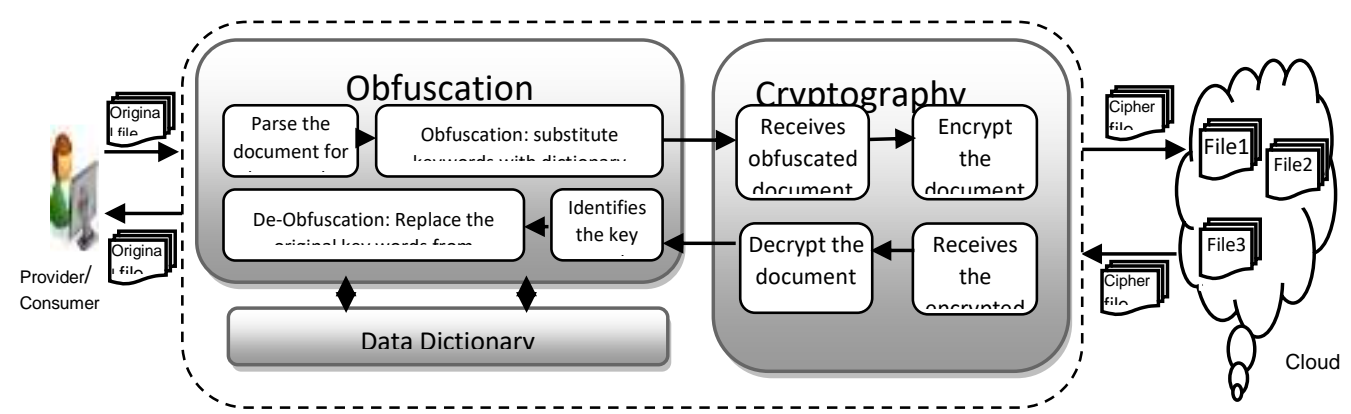

Fig. 1 Structure of proposed algorithm

The proposed algorithm has three modules, Obfuscator and Cryptanalysis module.

Obfuscator: This module performs the obfuscation and de-obfuscation operations on input document that contains structured or unstructured data. Whenever a customer wants to store his confidential information in cloud, the obfuscator module obfuscate the document and sends it to cryptanalysis module. And de-obfuscate the document, whenever customer wants to use it. This module attempts to retains the readability of the original document, and scrambles the sensitive information in the original document is such a manner that it is possible to reconstruct the original document from the obfuscated document [28][29].

Cryptanalysis: Usually cryptanalysis module transforms the information in to a form that can't be read and understand by other users who is not authorized. It takes the obfuscated document and encrypt it by using a public key encryption algorithm RSA which can be implemented practically and yields better results than rest of the algorithms [31][32]. The workflow algorithms are shown in Figure 2.

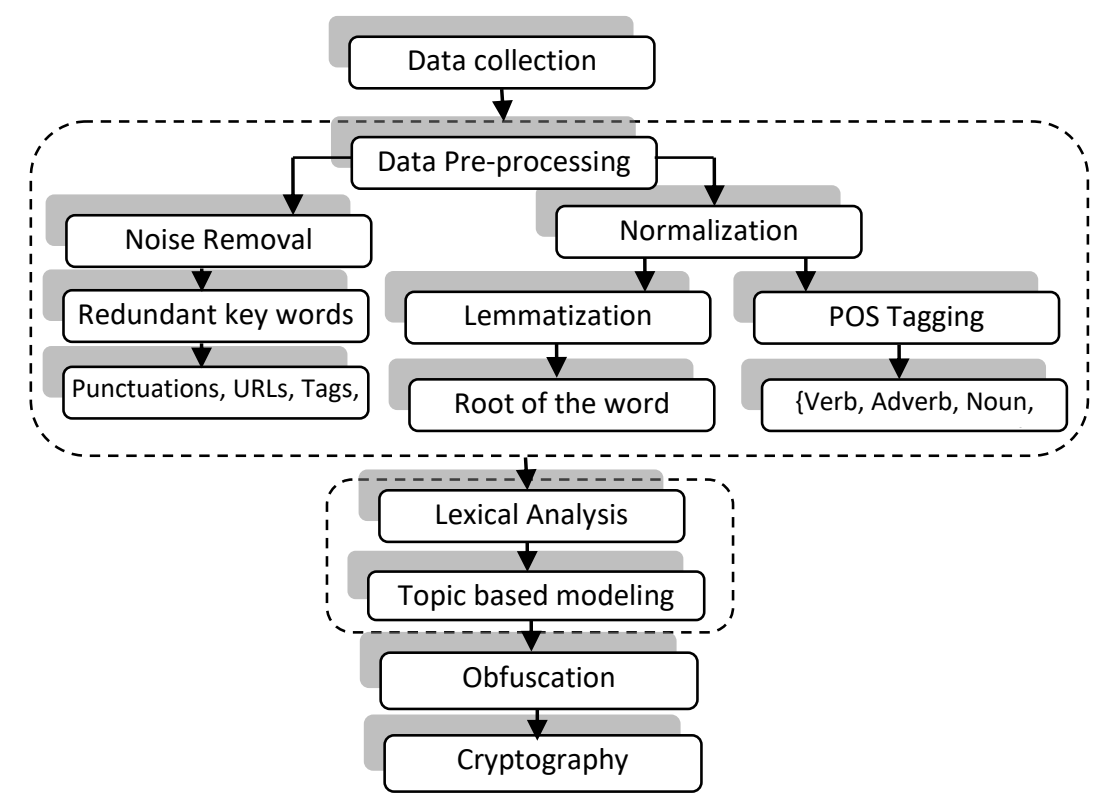

Fig. 2 Workflow of Algorithm

In Figure 2, initially the sample data is collected for obfuscation. To extract the key words from document we perform the following steps.

Data Pre-processing: Data pre-processing includes two steps they are (i) Noise removal and (ii) Normalization. Noise Removal: Various data pre-processing techniques have been applied on the data for noise removal. 
Techniques have been used to remove the redundant key words, punctuations, URLs, tags, and stop words etc., [27]. Stop words refer to large number of prepositions, which are to be filtered out before processing the data.

Normalization: Normalization is a process of converting a list of words to a more uniform sequence. This is useful in preparing text for later processing. Techniques like converting text in to lower case, parts of speech (POS) tagging, lemmatization has been applied on the data for smooth processing. Lemmatization and POS tagging are the special cases of normalization [30].

Lemmatization: Lemmatization is a more advanced technique which works based on the root of the word.

POS tagging is the process of marking up a word in a corpus to a corresponding part of a speech tag, based on its context and definition. As a particular word may have a different part of speech based on the context in which the word is used. POS Tagging is a very important step to understand the meaning of any sentence or to extract relationships and build a knowledge graph. Python NLP package is used for implementing the techniques.

Lexical Analysis: It involves identifying and analyzing the structure of words. Lexicon of a language means the collection of words and phrases in a language. Lexical analysis is dividing the whole chunk of text into paragraphs, sentences, and words [31].

Topic based modelling: Topic vocabulary has been built using Latent Dirichlet Allocation (LDA) model. Based on the number of topics constructed from LDA, a set of seed words will be extracted for building the Topic-Based Modeling.

A set of words for each topic can be extracted by using the below equation 1 .

$$
P\left(\theta_{1: M}, Z_{1, M} \beta_{1: K} / D ; \alpha_{1: M}, \eta_{1: K}\right) \rightarrow \text { (1) }
$$

Where ${ }^{\prime} \theta^{T}$ denote the distribution of topics, ${ }^{r} z^{g}$ number of topics for each document, ${ }^{\prime} \beta^{\prime}$ denotes the distribution of words for each topic. Given ${ }^{\prime} D^{s}$ denotes the document, ${ }^{\prime} \alpha^{s}$ and ${ }^{\prime} \eta$ 'denotes the parameter vectors for document and topic respectively.

But this can't be calculated nicely because this entity is intractable. To solve this problem, variational inference technique have been used to minimize the KL divergence between the approximation and true posterior as an optimization problem using the equation 2 .

$$
\gamma^{*}, \emptyset^{*}, \lambda^{*}=\operatorname{argmin}_{(\gamma, \phi, \lambda)} D(q(\theta, Z, \beta / \gamma, \phi, \lambda) / / p(\theta, z, \beta / D ; \alpha, \eta) \rightarrow(2)
$$

Where $\gamma, \phi$ and $\lambda$ represent the free variational parameters we approximate $\theta, \mathbf{z}$ and $\beta$ with, respectively. Here $\mathrm{D}(\mathrm{q} \| \mathrm{p})$ represents the $K L$ divergence between $\mathrm{q}$ and $\mathrm{p}$. And by changing $\gamma, \phi$ and $\lambda$, we get different $\mathrm{q}$ distributions having different distances from the true posterior p. Our goal is to find the $\gamma^{*}, \phi^{*}$ and $\lambda^{*}$ that minimize the $K L$ divergence between the approximation $\mathrm{q}$ and the true posterior $\mathrm{p}$.

Obfuscation: Data obfuscation is a form of data masking where data is purposely scrambled/replaced with dummy data to prevent unauthorized access to sensitive materials [18]. This form of scrambling/replacement results in unintelligible or confusing data. Data obfuscation at this point scrambles the data in a manner that retains readability. Hence we have two advantages (i) when third party (illegitimate user) get access to document, he can't conclude which data is original and which is replaced, (ii) since enough information available, we can perform analytical and processing application on document. All the key words which are extracted in the previous step would be replaced with key words in the custom built dictionary by applying "FlashText algorithm". Below 
Figure 3 shows obfuscation of record 2, in which original information is replaced with dummy information.

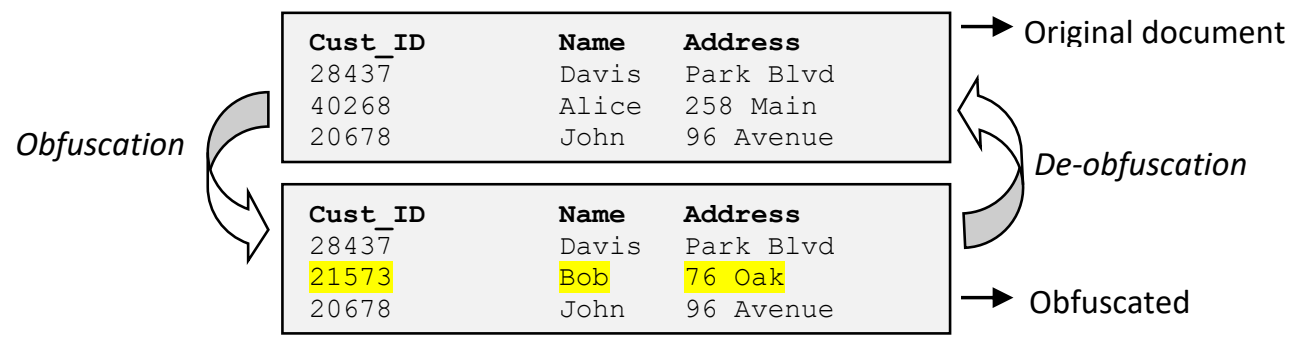

Fig. 3 Data obfuscation

Cryptography: Is a process of keeping a information secret. i.e it transforms the information in to a form that can't be read and understand by other users who is not authorized. It has four concerns, confidentiality, integrity, non-repudiation and authentication. In our work we use cryptography technique to ensure the confidentiality information stored in cloud [33][34].

At this level, the obfuscated document will be encrypted by using a public key encryption algorithm RSA which can be implemented practically and yields better results than rest of the algorithms. The working procedure of RSA is given below.

The encryption and decryption process is done using the equations $4 \& 5$.

$$
\begin{aligned}
& C=M^{n} e \bmod n \rightarrow(4) \\
& M=C^{n} d \bmod n \rightarrow(5)
\end{aligned}
$$

Where $C$ and $M$ are the cipher text and Plain text.

Here the value of ${ }^{n}$, should be known to the cloud service provider as well as the cloud service consumer who wants to use the data. And service provider knows the value of ' $e$, and only authorized consumer knows the value of ${ }^{d} d$. Here public key is $\left\{{ }^{e},{ }^{n}\right\}$ and private key is $\left\{{ }^{d},{ }^{n}\right\}$.

And, to determine the values of ${ }^{e},{ }^{d},{ }^{n}$ we have to follow the steps below.

Step-1: Choose two numbers ${ }^{p}$ and ${ }^{q}$ which are huge primes.

Step-2: Calculate $\mathrm{n}$ as the multiplication of ${ }^{p}$ and ${ }^{q}$.

Step-3: Choose some large integer randomly, $d$, such that $\operatorname{GCD}\left(d_{s}((p-1) *(q-1))\right)=1$.

Step-4: Find the value of ${ }^{\prime{ }^{\prime}}$ such that ${ }^{e * d}=1(\bmod ((p-1) *(q-1)))$.

Based on these values and following the RSA procedure the input gets converted to cipher text.

\section{EXPERIMENTAL RESULTS AND ITS ANALYSIS}

The proposed scheme is implemented on CloudSim and performance of the same is verified with existing techniques in terms of time taken for both obfuscation/encryption and decryption/deobfuscation. The experimental results show that security level of proposed scheme is higher than existing techniques. The existing technique works on 
structured numerical data whereas proposed scheme works effectively on unstructured data.

The efficiency of the proposed scheme lies in exact keywords extraction based on semantics, substituting keywords with dummy keywords (obfuscation) and substitution of dummy keywords with original keywords (de-obfuscation). The process of substituting the all original keywords with dummy keywords and dummy keywords with exact original keywords without any keyword missing.

Table I. Time for keyword extraction, obfuscation and de-obfuscation \& percentage of keyword hit

\begin{tabular}{|c|c|c|c|c|c|c|}
\hline $\begin{array}{c}\text { File size } \\
\text { (Kb) }\end{array}$ & $\begin{array}{c}\text { No. of of } \\
\text { topics }\end{array}$ & $\begin{array}{c}\text { No. of } \\
\text { key } \\
\text { words }\end{array}$ & $\begin{array}{c}\text { Time } \\
\text { taken for } \\
\text { keywords } \\
\text { extraction } \\
\text { (MilliSec) }\end{array}$ & $\begin{array}{c}\text { \% } \\
\text { Keyword } \\
\text { hit }\end{array}$ & $\begin{array}{c}\text { Time for } \\
\text { obfuscation }\end{array}$ & $\begin{array}{c}\text { Time for } \\
\text { de- } \\
\text { obfuscation }\end{array}$ \\
\hline $1 \mathrm{~Kb}$ & 2 & 10 & 21 & $100 \%$ & 42 & 42 \\
\hline $10 \mathrm{~Kb}$ & 20 & 100 & 48 & $100 \%$ & 65 & 65 \\
\hline $50 \mathrm{~Kb}$ & 100 & 215 & 63 & $100 \%$ & 87 & 87 \\
\hline $100 \mathrm{~Kb}$ & 200 & 450 & 82 & $100 \%$ & 101 & 101 \\
\hline $200 \mathrm{~Kb}$ & 400 & 923 & 97 & $100 \%$ & 134 & 134 \\
\hline $400 \mathrm{~Kb}$ & 800 & 1765 & 221 & $100 \%$ & 234 & 234 \\
\hline $600 \mathrm{~Kb}$ & 1200 & 2534 & 325 & $100 \%$ & 423 & 423 \\
\hline $800 \mathrm{~Kb}$ & 1600 & 3324 & 487 & $100 \%$ & 512 & 512 \\
\hline $1000 \mathrm{~kb}$ & 2000 & 4312 & 561 & $100 \%$ & 637 & 637 \\
\hline
\end{tabular}

Table II. Processing time of existing and proposed techniques for encryption and obfuscation

\begin{tabular}{|c|c|c|}
\hline File Size (Kb) & $\begin{array}{c}\text { Existing Scheme } \\
\text { (MilliSec) }\end{array}$ & $\begin{array}{l}\text { Proposed scheme } \\
\text { (MilliSec) }\end{array}$ \\
\hline $1 \mathrm{~Kb}$ & 225 & 211 \\
\hline $10 \mathrm{~Kb}$ & 452 & 412 \\
\hline $50 \mathrm{~Kb}$ & 641 & 627 \\
\hline $100 \mathrm{~Kb}$ & 873 & 848 \\
\hline $200 \mathrm{~Kb}$ & 989 & 956 \\
\hline $400 \mathrm{~Kb}$ & 2239 & 2198 \\
\hline $600 \mathrm{~Kb}$ & 3374 & 3211 \\
\hline $800 \mathrm{~Kb}$ & 4726 & 4613 \\
\hline $1000 \mathrm{~kb}$ & 5382 & 5123 \\
\hline
\end{tabular}

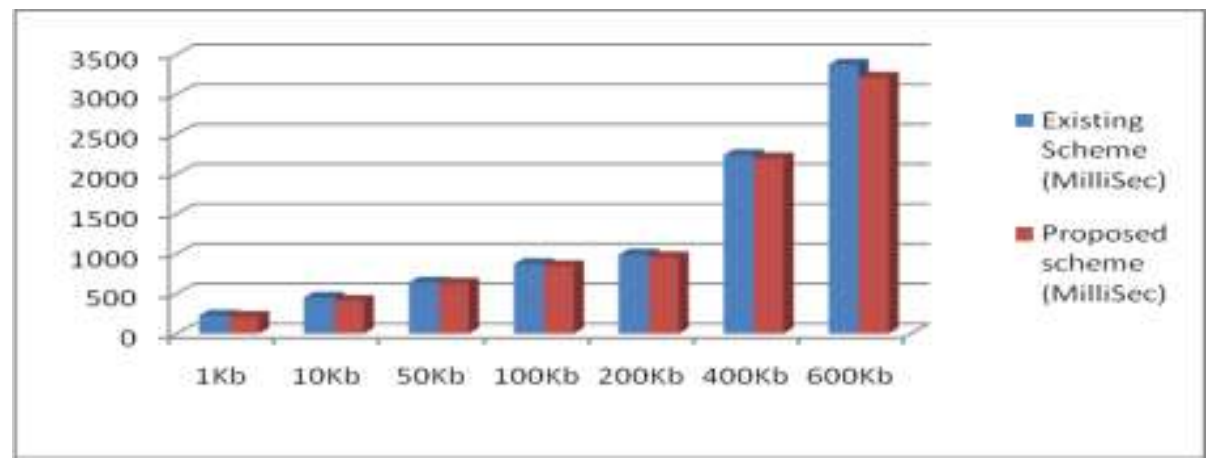

Fig. 7 Comparison between existing and proposed techniques processing time for encryption and obfuscation 
Figure7 shows that the proposed scheme takes less time for both obfuscation and encryption when compared with the existing scheme.

Table III. Processing time of existing and proposed techniques for decryption and deobfuscation

\begin{tabular}{|c|c|c|}
\hline $\begin{array}{c}\text { File Size } \\
(\mathbf{K b})\end{array}$ & $\begin{array}{c}\text { Existing Scheme } \\
\text { (MilliSec) }\end{array}$ & $\begin{array}{c}\text { Proposed scheme } \\
\text { (MilliSec) }\end{array}$ \\
\hline $1 \mathrm{~Kb}$ & 232 & 224 \\
\hline $10 \mathrm{~Kb}$ & 434 & 412 \\
\hline $50 \mathrm{~Kb}$ & 623 & 602 \\
\hline $100 \mathrm{~Kb}$ & 840 & 824 \\
\hline $200 \mathrm{~Kb}$ & 1063 & 1003 \\
\hline $400 \mathrm{~Kb}$ & 2212 & 2201 \\
\hline $600 \mathrm{~Kb}$ & 3339 & 3256 \\
\hline $800 \mathrm{~Kb}$ & 4726 & 4613 \\
\hline $1000 \mathrm{~kb}$ & 5382 & 5123 \\
\hline
\end{tabular}

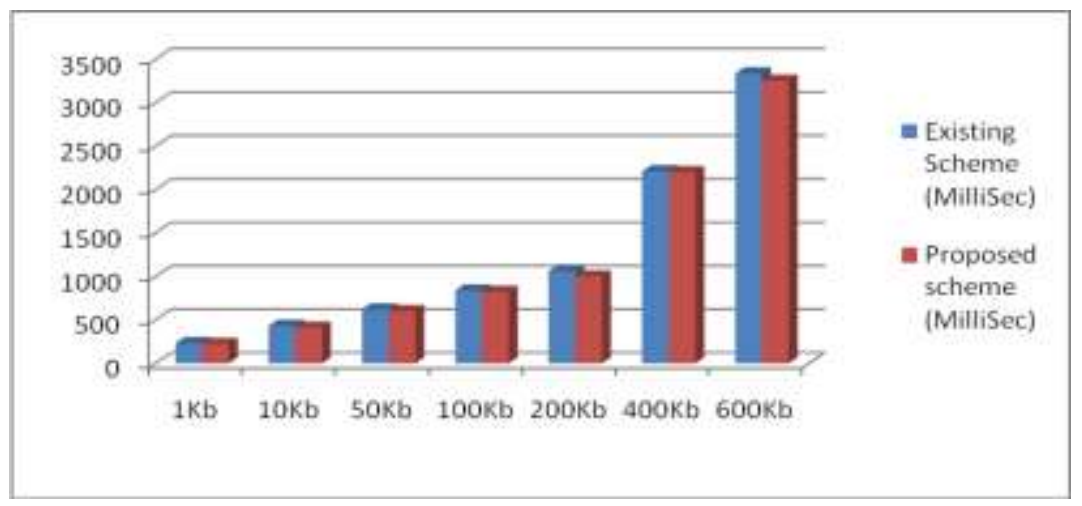

Fig. 8 Comparison between existing and proposed techniques processing time for decryption and de-obfuscation

Figure 8 shows that the proposed scheme takes less time for both obfuscation and encryption when compared with the existing scheme.

Table IV. Level of security offered by existing and proposed schemes

\begin{tabular}{|c|c|}
\hline Crypto Techniques & Security level \\
\hline Existing scheme & 93 \\
\hline Proposed scheme & 98 \\
\hline
\end{tabular}

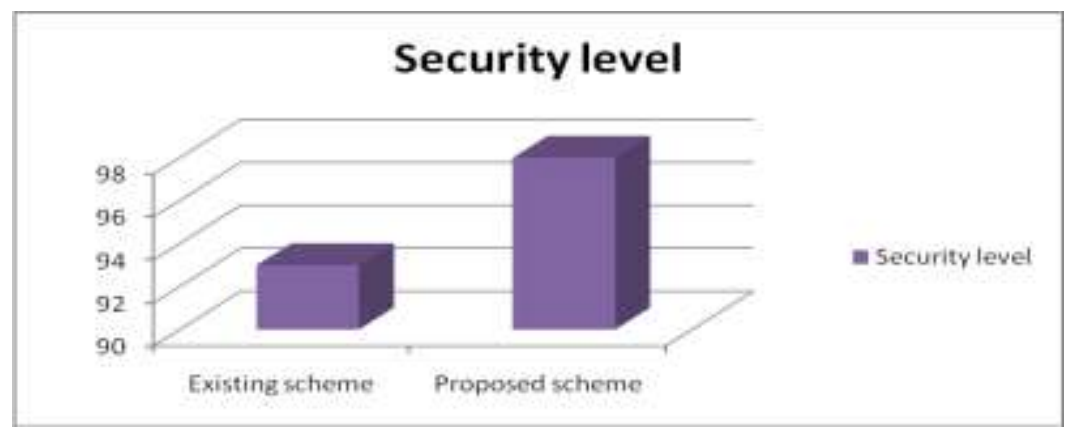

Fig. 9 Comparison between security levels of existing and proposed schemes 
Figure 9 shows that the security level offered by the proposed scheme is much higher than existing scheme.

\section{CONCLUSION}

This work is aimed to propose an efficient algorithm with obfuscation and cryptography for unstructured data, which preserve confidentiality of data stored in cloud at two levels. At the fist level the algorithm obfuscate the document by replacing the key words (obfuscation), and at the second level obfuscated document is encrypted using traditional RSA algorithm for better security. The efficiency of the proposed scheme lies in exact keywords extraction based on semantics, substituting keywords with dummy keywords (obfuscation) and later dummy keywords with original keywords (deobfuscation), also time taken for obfuscation and de-obfuscation. The proposed scheme is implemented on CloudSim and performance of the same is verified with existing techniques in terms of time taken for both obfuscation/encryption and decryption/deobfuscation. The experimental results show that security level of proposed scheme is higher than existing techniques.

\section{REFERENCES}

[1] M. T. Khorshed, A. B. M. S. Ali, and S. A. Wasimi, "A survey on gaps, threat remediation challenges and some thoughts for proactive attack detection in cloud computing," Futur. Gener. Comput. Syst., vol. 28, no. 6, pp. 833-851, 2012.

[2] M. Pathan, C. Vecchiola, and R. Buyya, "Load and proximity aware request-redirection for dynamic load distribution in peering CDNs," Lect. Notes Comput. Sci. (including Subser. Lect. Notes Artif. Intell. Lect. Notes Bioinformatics), vol. 5331 LNCS, no. PART 1, pp. 62-81, 2008.

[3] T. Halabi and M. Bellaiche, "A broker-based framework for standardization and management of Cloud Security-SLAs," Comput. Secur., vol. 75, pp. 59-71, 2018.

[4] E. Deepak Chowdary and D. Yakobu, "Cloud of Things (CoT) integration challenges," 2016 IEEE Int. Conf. Comput. Intell. Comput. Res. ICCIC 2016, 2017.

[5] S. S. Chauhan, E. S. Pilli, R. C. Joshi, G. Singh, and M. C. Govil, "Brokering in interconnected cloud computing environments: A survey," J. Parallel Distrib. Comput., 2018.

[6] A. J. Ferrer, "Inter-cloud Research: Vision for 2020," Procedia Comput. Sci., vol. 97, pp. 140-143, 2016.

[7] S. Asad, M. Fatima, A. Saeed, and I. Raza, "Multilevel classification of security concerns in cloud computing," Appl. Comput. Informatics, vol. 13, no. 1, pp. 57-65, 2017.

[8] Y. Sun, J. Zhang, Y. Xiong, and G. Zhu, "Data Security and Privacy in Cloud Computing," vol. 2014, 2014.

[9] B. T. Rao, “A Study on Data Storage Security Issues in Cloud Computing," Procedia - Procedia Comput. Sci., vol. 92, pp. 128-135, 2016.

[10] R. Wang, "Research on data security technology based on cloud storage," Procedia Eng., vol. 174, pp. 1340-1355, 2017.

[11] N. Hidayah, A. Rahman, and K. R. Choo, "ScienceDirect A survey of information security incident handling in the cloud," Comput. Secur., vol. 49, pp. 45-69, 2014.

[12] S. Singh, Y. Jeong, and J. Hyuk, "Author' s Accepted Manuscript A Survey on Cloud Computing Security: Issues, Threats , and Solutions,” J. Netw. Comput. Appl., 2016.

[13] R. Bhadauria, "Survey on Security Issues in Cloud Computing and Associated Mitigation Techniques."

[14] M. Usman, M. Ahmad, and X. He, "Cryptography-based secure data storage and sharing using HEVC and public clouds,” Inf. Sci. (Ny)., vol. 387, no. 2017, pp. 90-102, 2019.

[15] M. Vijayalakshmi, D. Yakobu, D. Veeraiah, and N. G. Rao, "Automatic healing of services in cloud computing environment," Proc. 2016 Int. Conf. Adv. Commun. Control Comput. Technol. ICACCCT 2016, no. 978, pp. 740-745, 2017.

[16] Gowthami Garikapati, Yakobu D, "An Analysis of Cloud Data Security Issues Mechanisms" “ijpam.eu," vol. 116, no. 6, pp. 141-147, 2017.

[17] C. Systems, "A Hybrid Secure Mechanism for Effective Storage Confidentiality to Ensure Data Integrity and Privacy in Public Cloud," vol. 11, pp. 330-339, 2019.

[18] B. Hashemzade and A. Maroosi, "Hybrid Obfuscation Using Signals and Encryption," J. Comput. Networks Commun., vol. 2018, pp. 1-6, 2018.

[19] N. Jorstad and T. Landgrave, "Cryptographic algorithm metrics," ... 20th Natl. Inf. Syst. ..., 1997.

[20] A. Ghosh and A. Saha, "A N UMERICAL M ETHOD B ASED E NCRYPTION," pp. 149-157, 2013.

[21] M. Horvath and L. Butty, "The Birth of Cryptographic Obfuscation - A Survey -," vol. 116675, no. 
116675, pp. 1-59, 2018.

[22] T. S. Murthy, N. P. Gopalan, and D. Yakobu, "An Efficient un-realization algorithm for privacy preserving decision tree learning using McDiarmid 's bound," no. 4, pp. 499-502.

[23] P. Examiner and A. W. Johns, “(12) United States Patent,” vol. 2, no. 12, 2010.

[24] L. H. I. B, F. Giunchiglia, L. A. Martucci, and R. Chenu-abente, "Privacy and Identity Management. Time for a Revolution?," vol. 476, no. 600854, pp. 343-358, 2016.

[25] S. Arul Oli and Dr. L. Arockiam, "Confidentiality Technique for Data Stored in Public Cloud Storage," Int. J. Eng. Res., vol. V5, no. 02, pp. 169-174, 2016.

[26] K. Bock and S. M. Garnsey, "Language Processing," A Companion to Cogn. Sci., pp. 226-234, 2008.

[27] N. A. Lewinski and B. T. McInnes, "Using natural language processing techniques to inform research on nanotechnology," Beilstein J. Nanotechnol., vol. 6, no. 1, pp. 1439-1449, 2015.

[28] S. Hohenberger, G. N. Rothblum, A. Shelat, and V. Vaikuntanathan, "Securely obfuscating reencryption," J. Cryptol., vol. 24, no. 4, pp. 694-719, 2011.

[29] G. Ravikumar, "Design of data masking architecture and analysis of data masking techniques for testing," Int. J. Eng. Sci. Technol., vol. 3, no. 6, pp. 5150-5159, 2011.

[30] S. Sun, C. Luo, and J. Chen, "A review of natural language processing techniques for opinion mining systems," Inf. Fusion, vol. 36, pp. 10-25, 2017.

[31] M. Sevenster, J. Buurman, P. Liu, J. F. Peters, and P. J. Chang, "Natural language processing techniques for extracting and categorizing finding measurements in narrative radiology reports," Appl. Clin. Inform., vol. 6, no. 3, pp. 600-610, 2015.

[32] Vijaya Lakshmi Paruchuri. Data Confidentiality in Cloud using Encryption Algorithms. International Journal of Cloud-Computing and Super-Computing. Vol. 3. No. 2. Dec. 2016.GVPress. pp:718.http://dx.doi.org/10.21742/IJCS.2016.3.2.02.

[33] S. Suresh Babu and Chimili Ankapanaidu. New Proof Methods for Improving Privacy and Security by using Abe. International Journal of Cloud-Computing and Super-Computing. Vol. 3. No. 1. Jun. 2016.GVPress. pp:1-6.http://dx.doi.org/10.21742/IJCS.2016.3.1.01.

[34] Durga Bhavani Dasari.Cloud-Based Venue Recommendation Framework. International Journal of Cloud-Computing and Super-Computing. Vol. 4. No. 1. Jun. 2017.GVPress. pp:2126.http://dx.doi.org/10.21742/IJCS.2017.4.1.04.

[35] P. Harika, N.T hirupathi Rao.A Survey on Cloud Computing - Investigative Points, Confronts, Design and Applications. International Journal of Cloud-Computing and Super-Computing. Vol. 5. No. 1. Jun. 2018.GVPress. pp:9-16.http://dx.doi.org/10.21742/IJCS.2018.5.1.02.

[36] Anil Bamwal and Asit Dwivedi. Effective Management of Security of Risk in Cloud Computing Environment. International Journal of Private Cloud Computing Environment and Management. Vol. 3. No. 1. Apr. 2016.GVPress. pp:1-10.http://dx.doi.org/10.21742/IJPCCEM.2016.3.1.01.

[37] S Rithika. Issues on Developing Interoperable Cloud Applications. International Journal of Private Cloud Computing Environment and Management. Vol. 4. No. 1. Apr. 2017.GVPress. pp:914.http://dx.doi.org/10.21742/IJPCCEM.2017.4.1.02

[38] K Praveen Kumar. Performing Initiative Data Prefetching in File Systems for Cloud Computing. International Journal of Private Cloud Computing Environment and Management. Vol. 4. No. 1. Apr. 2017.GVPress. pp:15-20.http://dx.doi.org/10.21742/IJPCCEM.2017.4.1.03.

[39] T. Ravi Kumar. Advanced Approach and Management in Cloud Trust. International Journal of Urban Design for Ubiquitous Computing. Vol. 4. No. 2. Sep. 2016.GVPress. pp:712.http://dx.doi.org/10.21742/IJUDUC.2016.4.2.02.

[40] Deduplicating Data in Cloud. International Journal of Advanced Research in Big Data Management System. Vol. 1. No. 1. Jun. 2017.GVPress. pp:31-38.http://dx.doi.org/10.21742/IJARBMS.2017.1.1.04.

[41] N.S.P. Bhargavi. Service Scheme for Data Cloud Services. International Journal of Advanced Research in Big Data Management System. Vol. 1. No. 1. Jun. 2017.GVPress. pp:4550.http://dx.doi.org/10.21742/IJARBMS.2017.1.1.06

[42] D. Yakobu, Hemanth Kumar Kalluri, Venkatesulu Dondeti, "An Enhanced Secure, Robust and Efficient CryptoScheme for Ensuring Data Privacy in Public Cloud using Obfuscation \& Encryption ", Nov. 2019, https://doi.org/10.18280/isi. 